\title{
Variability in Global Top-of-Atmosphere Shortwave Radiation Between 2000 and 2005
}

\author{
Norman G. Loeb ${ }^{1}$, Bruce A. Wielicki \\ NASA Langley Research Center, Hampton University, Hampton, Virginia \\ Fred G. Rose, David R. Doelling \\ Analytical Services \& Materials Inc., Hampton, Virginia
}

Geophysical Research Letters

Submitted September, 2006

\footnotetext{
${ }^{1}$ Corresponding Author Address: Dr. Norman G. Loeb, Mail Stop 420, NASA Langley

Research Center, Hampton, VA 23681-2199, U.S.A. (757) 864-5688 (office); (757) 8647996 (fax);

Email: n.g.loeb@larc.nasa.gov
} 


\section{Abstract}

Measurements from various instruments and analysis techniques are used to

2 directly compare changes in Earth-atmosphere shortwave (SW) top-of-atmosphere (TOA)

3 radiation between 2000 and 2005. Included in the comparison are estimates of TOA

4 reflectance variability from published ground-based Earthshine observations and from

5 new satellite-based CERES, MODIS and ISCCP results. The ground-based Earthshine

6 data show an order-of-magnitude more variability in annual mean SW TOA flux than

7 either CERES or ISCCP, while ISCCP and CERES SW TOA flux variability is consistent

8 to $40 \%$. Most of the variability in CERES TOA flux is shown to be dominated by

9 variations global cloud fraction, as observed using coincident CERES and MODIS data.

10 Idealized Earthshine simulations of TOA SW radiation variability for a lunar-based

11 observer show far less variability than the ground-based Earthshine observations, but are

12 still a factor of 4-5 times more variable than global CERES SW TOA flux results.

13 Furthermore, while CERES global albedos exhibit a well-defined seasonal cycle each

14 year, the seasonal cycle in the lunar Earthshine reflectance simulations is highly variable

15 and out-of-phase from one year to the next. Radiative transfer model (RTM) approaches

16 that use imager cloud and aerosol retrievals reproduce most of the change in SW TOA

17 radiation observed in broadband CERES data. However, assumptions used to represent

18 the spectral properties of the atmosphere, clouds, aerosols and surface in the RTM

19 calculations can introduce significant uncertainties in annual mean changes in regional

20 and global SW TOA flux. 


\section{1. Introduction}

4 energy that drives the climate system. It is determined from the difference between how

5 much solar radiation is intercepted by the planet and how much is reflected back to space.

6 Over a year, a planetary radiation balance is approached whereby the absorbed solar

7 radiation is nearly balanced by outgoing terrestrial infrared radiation. A change in

8 planetary albedo could significantly modify this balance and alter climate. For example,

9 an increase in albedo would mean less solar heating and offset the influence of increased

10 absorption of infrared radiation in the atmosphere due to the buildup of $\mathrm{CO} 2$, $\mathrm{CH} 4$, and

11 other greenhouse gases. Sensitivity studies show that an absolute increase (decrease) in

12 planetary albedo of 0.01 could potentially lead to a decrease (increase) in equilibrium

13 surface temperature by as much as $1.75^{\circ} \mathrm{C}$ (Cess, 1976).

15 in planetary albedo during the first part of the $21^{\text {st }}$ century. Using ground-based

16 measurements of Earthshine, Pallé et al. (2004) find that the Earth’s reflectance has

17 increased by as much as 0.018 between 1999 and 2003, corresponding to a change of 6

$18 \mathrm{Wm}^{-2}$ in top-of-atmosphere (TOA) radiative flux $(\Delta F=\Delta \alpha \times C / 4$, where $F=\mathrm{TOA}$ flux,

$19 \alpha=$ =albedo, $C=1365 \mathrm{Wm}^{-2}$ ). Pallé et al. (2004) estimate the Earth’s albedo using telescope

20 measurements of the visible solar radiation that is first reflected by the Earth towards the

21 Moon and then back from the Moon to an observer on the night-side of the Earth at the

22 Big Bear Solar Observatory (BBSO) in California. The ground-based Earthshine

23 measurements (Pallé et al., 2004) sample only 1/3 of the Earth on any given day, and

24 cover at most $\sim 1 / 3$ of each lunar month provided the night-sky is cloud-free. A $6 \mathrm{Wm}^{-2}$ 
1 change is climatologically significant as it exceeds the radiative forcing caused by Mount

2 Pinatubo in the early 1990s by a factor of 2.5, and is twice as large as the longwave

3 radiative forcing by greenhouse gases since 1850 (Houghton et al., 2001). Pallé et al.

4 (2004) find support for this result from variations in the satellite on-board temperatures of

5 the Global Ozone Monitoring Experiment (GOME) instrument, which are hypothesized

6 to be influenced by changes in Earth's outgoing radiance (Casadio et al., 2005). In

7 contrast, the global CERES observations show a small decrease of $\sim 2 \mathrm{Wm}^{-2}$ in shortwave

8 reflected flux between 2000 and 2004 (Wielicki et al., 2005). More recently, Loeb et al.

9 (2006) used a revised version of the CERES data to show that no statistically significant

10 changes in the Earth's albedo have occurred between 2000 and 2005. Their results are

11 consistent with changes in Photosynthetically Active Radiation (PAR) during the same

12 period from Sea-Viewing Wide-Field-of-View Sensor (SeaWiFS) TOA radiance

13 measurements (Patt et al., 2003) for all-sky conditions over ocean. PAR is defined as the

14 solar flux reaching the ocean surface in the $400-700 \mathrm{~nm}$ spectral range and is anti-

15 correlated to SW TOA flux. Loeb et al. (2006) also found consistent results between

16 CERES monthly SW TOA flux anomalies and those from the International Satellite

17 Cloud Climatology Project (ISCCP) radiative flux profile data set (ISCCP-FD product)

18 (Zhang et al., 2004).

19 In this study, several different approaches for monitoring the variability in the

20 Earth’s TOA SW Radiation are considered. In Section 2 we perform simulations of lunar-

21 based Earthshine measurements in order to assess whether or not this approach is suitable

22 for monitoring changes in the Earth's albedo. In Section 3 we compare temporal changes

23 in TOA SW radiation using radiative transfer models initialized with retrievals from the 
1 Moderate Resolution Imaging Spectroradiometer (MODIS) with coincident radiative

2 fluxes obtained directly from CERES broadband measurements. Finally, Section 4

3 compares annual anomalies in SW TOA flux from ground-based Earthshine, CERES and

4 ISCCP between 2000 and 2005, as well as monthly anomalies from CERES SW TOA

5 flux and MODIS cloud fraction.

\section{2. Seasonal Cycle of Albedo}

Global albedo has a distinct and repeatable seasonal cycle. Because of the tilt of

8 the Earth's axis and the large surface albedo of snow at high latitudes, it reaches

9 maximum values during the solstice months and minimum values during the equinox

10 months. Changes in cloud and surface properties from year to year introduce slight

11 variations in the seasonal cycle of albedo, but these perturbations are much smaller than

12 the basic seasonal cycle of albedo. As a minimum, therefore, any approach that attempts

13 to measure subtle changes in the Earth's albedo must be able to characterize the broad

14 features of global albedo changes with season.

15 Here we simulate the seasonal cycle of the Earth's reflectance as it would appear

16 from an observer on the moon viewing Earthshine. The simulation uses CERES Angular

17 Distribution Models (ADMs) (Loeb et al., 2003) and measurements from CERES Terra

18 Single Satellite Footprint (SSF) product for 2002 through 2004. Assuming no changes in

19 scene properties between the CERES and Earthshine measurement times, we can

20 simulate the reflectance contributions from all points on the Earth that contribute to

21 Earthshine by using the CERES ADMs to transform the observed reflectance at the

22 CERES viewing geometry to that corresponding to the sun-earth-moon viewing geometry

23 at the Earthshine observation time. These reflectances are then integrated over the lunar 
1 viewing geometry to determine the total Earthshine reflectance intercepted by the moon.

2 Conceptually, we can think of the moon as a satellite measuring the Earth's reflectance

3 over a large portion of the Earth from a viewing geometry defined by the relative position

4 of the sun, earth and moon. To calculate the sun-earth-moon geometry, the Science Data

5 Production Toolkit for the Earth Observing System Data and Information System

6 (EOSDIS) Core System (ECS) is used. For each day, the Earthshine simulation is

7 updated hourly. Following Pallé et al. (2004), only observations with a lunar phase angle

8 between $65^{\circ}$ and $135^{\circ}$ are considered. To remove reflectance variations due to changes in

9 lunar viewing geometry during the lunar month, the reflectance at every time-step is

10 adjusted to a common lunar phase angle of $95^{\circ}$ using a $5^{\text {th }}$-order polynomial regression fit

11 of Earthshine reflectance against lunar phase angle for data between 2002 and 2004.

12 Because the lunar observer is assumed to collect data continuously throughout the day

13 from all parts of the Earth that contribute to Earthshine, the spatial and temporal sampling

14 in this simulation is more complete than the ground-based Earthshine measurements of

15 Pallé et al. (2004), which are restricted to a few hours per night and limited to cloud-free

16 conditions at one site (BBSO). The lunar Earthshine simulation represents ideal sampling

17 conditions: it is analogous to having multiple ground-based Earthshine sites around the

18 globe with no intercalibration differences, no cloud coverage restrictions, and since

19 CERES SW data are considered, no narrow-to-broadband conversion errors.

20 Because of Terra's sun-synchronous $98^{\circ}$ inclination orbit and the wide swath

21 width of CERES, CERES acquires global coverage on a daily basis. The first step to

22 determining global albedo from CERES Terra radiance measurements is to apply CERES

23 ADMs to convert measured radiances to radiative flux values (Loeb et al., 2005). Each 
1 instantaneous TOA flux is converted to a 24-hour TOA flux by applying diurnal albedo

2 models that account for albedo changes at all times of the day, assuming the scene at the

3 CERES Terra overpass time remains invariant throughout the day. This assumption is

4 found to have a negligible effect on the interannual variability of albedo at global scales.

5 The diurnal albedo models were derived from ADMs developed from CERES

6 measurements on the Tropical Rainfall Measuring Mission Satellite (TRMM) (Loeb et

7 al., 2003). TOA fluxes from each day are then placed on a $1^{\circ} \times 1^{\circ}$ latitude-longitude equal-

8 area nested grid and averaged over the month. A global monthly mean albedo is

9 determined from the ratio of the global monthly mean SW TOA flux to the global mean

10 solar insolation at the TOA for the month.

11 Fig. 1a-b compares the seasonal cycle in simulated Earthshine reflectance and

12 CERES global albedo given as the deviation from the mean 3-year value for 2002

13 through 2004. CERES global albedos exhibit a well-defined seasonal cycle with albedo

14 maxima and minima occurring at the same time of year for each of the 3 years. In

15 contrast, while a seasonal cycle in Earthshine reflectance is apparent in Fig. 1a, it is

16 highly variable and out-of-phase from one year to the next. As a result, the year-to-year

17 variability in a given month is typically 4-5 times larger for Earthshine reflectance

18 compared to CERES albedo.

19 3. Temporal Variations in TOA SW Radiation Using Radiative 20 Transfer Models

21 An alternate way of inferring broadband TOA albedo is through radiative transfer

22 model (RTM) calculations with input cloud, atmosphere and surface properties derived

23 from global measurements. This approach is used in the International Satellite Cloud

24 Climatology Project (ISCCP) (Rossow et al., 1999) to create the ISCCP-FD data product 
1 which contains global 280-km radiative fluxes at 3-hour time steps (Zhang et al., 2004).

2 The CERES project also provides radiative fluxes that are based on RTM calculations

3 with cloud and aerosol properties from MODIS measurements (Wielicki et al., 1996;

4 Charlock et al., 1997). This dataset, called the Clouds and Radiative Swath (CRS) data

5 product, provides TOA fluxes at the CERES footprint scale at the CERES time of

6 observation. Calculated radiative fluxes in the CRS product are independent of those in

7 the CERES SSF data product, which are inferred directly from CERES broadband

8 radiance measurements (Loeb et al., 2005). Since narrowband radiance measurements are

9 the primary inputs, the RTM approach involves an approximation of the spectral and

10 bidirectional properties of the atmosphere, clouds, aerosols and the surface over the

11 globe. In order to assess the suitability of the RTM approach to characterize temporal

12 variations in SW radiation, we compare the change in regional annual mean SW TOA

13 flux distribution between 2004 and 2001 based on the RTM approach with that based on

14 radiative fluxes from CERES SSF product using the following expression:

$$
\Delta F_{04-01}(\lambda, \phi)=\Delta F_{04-01}^{C R S}(\lambda, \phi)-\Delta F_{04-01}^{S S F}(\lambda, \phi)
$$

16 where $\Delta F_{04-01}^{C R S}$ represents the difference in annual mean SW TOA flux between 2004 and

172001 in the CRS data product, $\Delta F_{04-01}^{S S F}$ is the corresponding difference from the SSF data

18 product, and $(\lambda, \phi)$ corresponds to the latitude-longitude region. $\Delta F_{04-01}$ is converted to

19 an equivalent 24-h average daily mean value by applying scaling factors derived from the

20 CERES Monthly TOA/Surface Averages (SRBAVG) and SSF data products. Results in

21 Fig. 2 show that the RTM approach underestimates the SW TOA flux change between

222004 and 2001 over land by up to $8 \mathrm{Wm}^{-2}$ (e.g., over central Asia), and overestimates the

23 flux change over the desert. Over ocean, the errors are smaller, but they exhibit a slight 
1 dependence upon latitude. Fig. 3 shows deseasonalized anomalies in the relative

2 difference between CRS and SSF reflected SW TOA flux for global ocean and land.

3 Anomalies in the relative difference generally remain within $\pm 1.5 \%$, and differ between

4 ocean and land by up to $1 \%$ in 2001. While fluctuations in CRS-SSF relative difference

5 anomalies with time are undoubtedly partly due to relative calibration changes between

6 CERES and MODIS (Loeb et al., 2006), the differences between ocean and land suggest

7 that narrow-to-broadband errors in the RTM approach (e.g., due to the assumed spectral

8 properties of the atmosphere, clouds, aerosols and surface) are also significant and can

9 introduce appreciable uncertainties in SW TOA flux changes.

\section{4. SW TOA Flux Anomalies}

11 An overall summary of annual anomalies in SW TOA flux between 2000 and

122004 based on several different methods is provided in Fig. 4a. The Earthshine BBSO

13 results are from Pallé et al. (2004), while "CERES global” results are based on global SW

14 TOA fluxes from the SSF data product that have been converted to 24-h average fluxes

15 by applying diurnal albedo models that account for albedo changes at all times of the day,

16 assuming the scene at the CERES Terra overpass time remains invariant throughout the

17 day (Loeb et al., 2006). ISCCP-FD global albedo anomalies are from Zhang et al. (2004),

18 while the "CERES Earthshine Simulation" results correspond to simulations described in

19 Section 2. Anomalies based on CERES CRS TOA fluxes are not included because

20 diurnal average fluxes based on instantaneous CRS TOA fluxes were unavailable at the

21 time of this study. The most striking feature in Fig. 4a is the marked difference between

22 Earthshine (BBSO) (Pallé et al., 2004) anomalies and all other methods. None of the

23 other methods support the dramatic increase in BBSO Earthshine reflectance between 
12000 and 2003. The standard deviation in the BBSO Earthshine anomalies is a factor of 5

2 greater than the simulated CERES Earthshine anomalies, and over an order-of-magnitude

3 greater than those from CERES global results. Interestingly, anomalies based on the

4 simulated CERES Earthshine approach bear little resemblance to the CERES global

5 results. In fact, the standard deviation in the simulated CERES Earthshine anomalies is a

6 factor of 2 larger than the CERES global results. While ISCCP and CERES SW TOA

7 flux anomalies also differ from one another from year to year, the standard deviation in

8 the anomalies is within $40 \%$ of each other.

9 Although anomalies based on CERES CRS TOA fluxes are not yet available for

10 comparison, it is possible to compare monthly anomalies in global mean CERES SW

11 TOA flux and MODIS cloud fraction, as shown in Fig. 4b. The strong correlation

12 between the anomalies in these two variables implies that most of the monthly variation

13 in SW TOA flux is associated with changes in cloud cover. The remaining variability is

14 likely associated with cloud optical depth variations and to a lesser extent surface albedo

15 variations.

\section{5. Conclusions}

17 Monitoring changes in the Earth's SW TOA radiation is critical for understanding

18 climate. However, because of the spatial and temporal scales involved and the need to

19 account for radiant energy across the entire solar spectrum, it presents a significant

20 observational challenge: all methods that attempt to measure the Earth's TOA radiation

21 suffer from sampling errors due to inadequate spatial, temporal or spectral coverage. This

22 study considers results from three independent approaches and compares recent changes

23 in TOA SW radiation between 2000 and 2005. The Earthshine ground-based approach of 
1 Pallé et al. (2004) shows the largest variability in annual mean SW TOA radiation,

2 reaching $6 \mathrm{Wm}^{-2}$ between 1999 and 2003. Simulations of lunar Earthshine measurements

3 show far less variability than those observed from the ground by Pallé et al. (2004), but

4 still show 4-5 times the variability observed from CERES global data. Furthermore, while

5 CERES global albedos exhibit a well-defined seasonal cycle with albedo maxima and

6 minima occurring at the same time each year, the seasonal cycle in lunar Earthshine

7 reflectance simulations is highly variable and out-of-phase from one year to the next.

8 Radiative transfer model (RTM) approaches that use imager cloud and aerosol retrievals

9 reproduce most of the change in SW TOA radiation observed in broadband CERES data,

10 but the assumptions used to represent the spectral properties of the atmosphere, clouds,

11 aerosols and surface in the RTM calculations can introduce significant uncertainties in

12 annual mean changes in regional and global SW TOA flux. Such uncertainties can cause

13 interannual anomaly errors ranging from $1 \%$ for global ocean to $2 \%$ for global land,

14 much too large for climate change decadal signals.

15 The largest uncertainty in global climate sensitivity over the next century is cloud

16 feedback. Since global cloud feedback has been shown to be linear in changing cloud

17 radiative forcing (CRF) (Soden and Held, 2006), this implies that changes in net CRF are

18 directly related to climate sensitivity. The difficulty in documenting climate variability

19 and change is in the calibration stability requirements. Estimates of anthropogenic total

20 radiative forcing in the next few decades are $0.6 \mathrm{Wm}^{-2}$ per decade (Houghton et al.,

21 2001). A $25 \%$ cloud feedback would change cloud net radiative forcing by $25 \%$ of the

22 anthropogenic radiative forcing, or $0.15 \mathrm{Wm}^{-2}$ per decade. The global average shortwave

23 (SW) or solar reflected cloud radiative forcing by clouds is $\sim 50 \mathrm{Wm}^{-2}$, so that the 
1 observation requirements for global broadband radiation budget to directly observe such

2 a cloud feedback is approximately $0.15 / 50=0.3 \%$ per decade in SW broadband

3 calibration stability (Ohring et al., 2005). Achieving this stability per decade in

4 calibration is extremely difficult and has only recently been demonstrated for the first

5 time by the ERBS and CERES broadband radiation budget instruments (Wong et al., 6 2006; Loeb et al., 2006). 


\section{Acknowledgements}

The CERES data are provided by the NASA Langley Atmospheric Sciences Data

Center in Hampton, Virginia. The ISCCP FD data set is obtained directly on-line from the ISCCP web site at isccp.giss.nasa.gov. The NASA Science Mission Directorate through the CERES project at Langley Research Center funded this study. 


\section{References}

Casadio, S., A. di Sarra, and G. Pisacane, 2005: Satellite on-board temperatures: Proxy measurements of Earth’s climate changes?, Geophys. Res. Lett., 32, L06704, doi:10.1029/2004GL022138.

Cess, R.D., 1976: Climate change: An appraisal of atmospheric feedback mechanisms employing zonal climatology, J. Atmos. Sci., 33, 1831-1843.

Charlock, T. P., F. G. Rose, D. A. Rutan, T. L. Alberta, D. P. Kratz, L.H. Coleman, G. L. Smith, N. Manalo-Smith, and T. D. Bess, 1997: Compute Surface and Atmospheric Fluxes (System 5.0), CERES Algorithm Theoretical Basis Document. 84 pp., [Available from http://asd-www.larc.nasa.gov/ATBD/pdf_docs/r2_2/ceres-atbd2.2s5.0.pdf].

Houghton et al., 2001: Intergovernmental Panel on Climate Change (IPCC), Climate Change 2001: The Scientific Basis. Contribution of Working Group I to the Third Assessment Report of the Intergovernmental Panel on Climate Change (IPCC), J. T. Houghton et al., Eds. (Cambridge Univ. Press, Cambridge, 2001).

Loeb, N. G., N. M. Smith, S. Kato, W. F. Miller, S. K. Gupta, P. Minnis, and B. A. Wielicki, 2003: Angular distribution models for top-of-atmosphere radiative flux estimation from the Clouds and the Earth's Radiant Energy System instrument on the Tropical Rainfall Measuring Mission Satellite. Part I: Methodology, J. Appl. Meteor., 42, $240-265$.

Loeb, N. G., S. Kato, K. Loukachine, and N. M. Smith, 2005: Angular distribution models for top-of-atmosphere radiative flux estimation from the Clouds and the 
Earth’s Radiant Energy System instrument on the Terra Satellite. Part I:

Methodology, J. Ocean and Atmos. Tech., 22, 338-351.

Loeb, N.G., B.A. Wielicki, W. Su, K. Loukachine, W. Sun, T. Wong, K.J. Priestley, G.

Matthews, W.F. Miller, and R. Davies, 2006: Multi-Instrument Comparison of Top of the Atmosphere Reflected Solar Radiation, J. Climate (in press).

Ohring, G., B. A. Wielicki, R. Spencer, B. Emery, and R. Datla, 2005: Satellite instrument calibration for measuring global climate change: Report of a Workshop, Bull. Amer. Met. Soc., 86, 1303-1313.

Pallé, E., P. R. Goode, P. Montañés-Rodriguez, and S. E. Koonin, 2004: Changes in the Earth's reflectance over the past two decades, Science, 304, 1299-1301, doi:10.1126/science.1094070.

Patt, F. S., R. A. Barnes, R. E. Eplee, Jr., B. A. Franz, W. D. Robinson, G. C. Feldman, S. W. Bailey, J. Gales, P. J. Werdell, M. Wang, R. Frouin, R. P. Stumpf, R. A. Arnone, R. W. Gould, Jr., P. M. Martinolich, V. Ransibrahmanakul, J. E. O'Reilly, and J. A. Yoder, 2003: Algorithm updates for the fourth SeaWiFS data reprocessing, NASA Tech. Memo. 2003--206892, 22, S.B. Hooker and E.R. Firestone, Eds., NASA Goddard Space Flight Center, Greenbelt, Maryland, 74 pp. [Available online from: http://oceancolor.gsfc.nasa.gov/cgi/postlaunch_tech_memo.pl?22]

Rossow, W. B., and R. A. Schiffer (1999), Advances in understanding clouds from ISCCP, Bull. Am. Meteorol. Soc., 80, 2261- 2287.

Soden, B.J., and I.M. Held, 2006: An assessment of Climate Feedbacks in Coupled Ocean-Atmosphere Models, J. Climate, 19, 3354-3360. 
Wielicki, B. A., B. R. Barkstrom, E. F. Harrison, R. B. Lee, G. L. Smith, and J. E. Cooper, 1996: Clouds and the Earth's Radiant Energy System (CERES): An Earth Observing System Experiment. Bull. Amer. Meteor. Soc., 77, 853-868.

Wielicki, B.A., T. Wong, N.G. Loeb, P. Minnis, K.J. Priestley, R. Kandel, 2005: Changes in Earth’s albedo measured by satellite, Science, 308, p. 825.

Wong, T., B. A. Wielicki, R. B. Lee, III, G. L. Smith, and K. A. Bush, 2006: Reexamination of the observed decadal variability of Earth radiation budget using altitude-corrected ERBE/ERBS Nonscanner WFOV data, J. Climate (in press).

Zhang, Y., W. B. Rossow, A. A. Lacis, V. Oinas, and M. I. Mishchenko (2004), Calculation of radiative fluxes from the surface to top of atmosphere based on ISCCP and other global data sets: Refinements of the radiative transfer model and the input data, J. Geophys. Res., 109, D19105, doi:10.1029/2003JD004457. 


\section{Figures}

Figure 1 Seasonal variation in (a) simulated Earthshine reflectance and (b) CERES global albedo expressed as the deviation from the 3-year mean value. Earthshine reflectances are adjusted to a common lunar phase angle of $95^{\circ}$.

Figure 2 Change in regional annual mean SW TOA flux distribution between 2004 and 2001 based on the RTM approach with that based on radiative fluxes from CERES SSF product.

Figure 3 Deseasonalized anomalies in the relative difference between CRS and SSF reflected SW TOA flux for global ocean and land from CERES and MODIS Terra observations.

Figure 4 (a) Global annual mean SW TOA flux anomalies from Earthshine BBSO (Palle et al., 2005), CERES Terra global fluxes, ISCCP-FD, and a simulation of Earthshine. (b) Monthly anomalies in global mean CERES SW TOA flux and MODIS cloud fraction. 

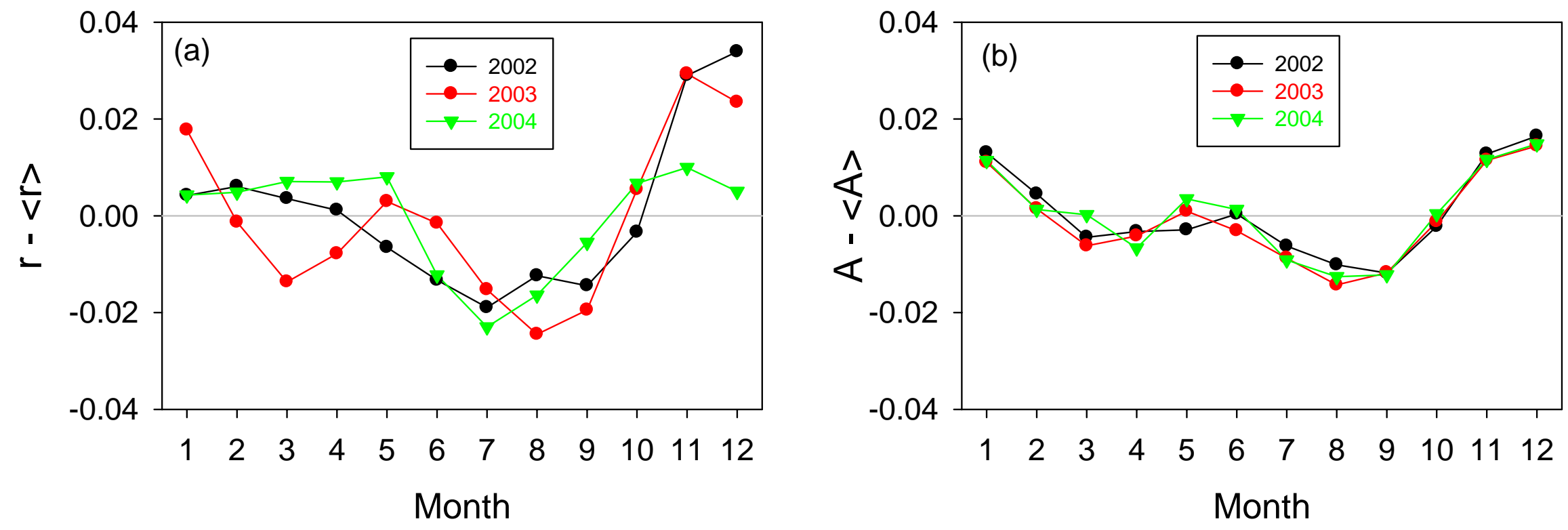

Figure 1 Seasonal variation in (a) simulated Earthshine reflectance and (b) CERES global albedo expressed as the deviation from the 3-year mean value. Earthshine reflectances are adjusted to a common lunar phase angle of $95^{\circ}$. 


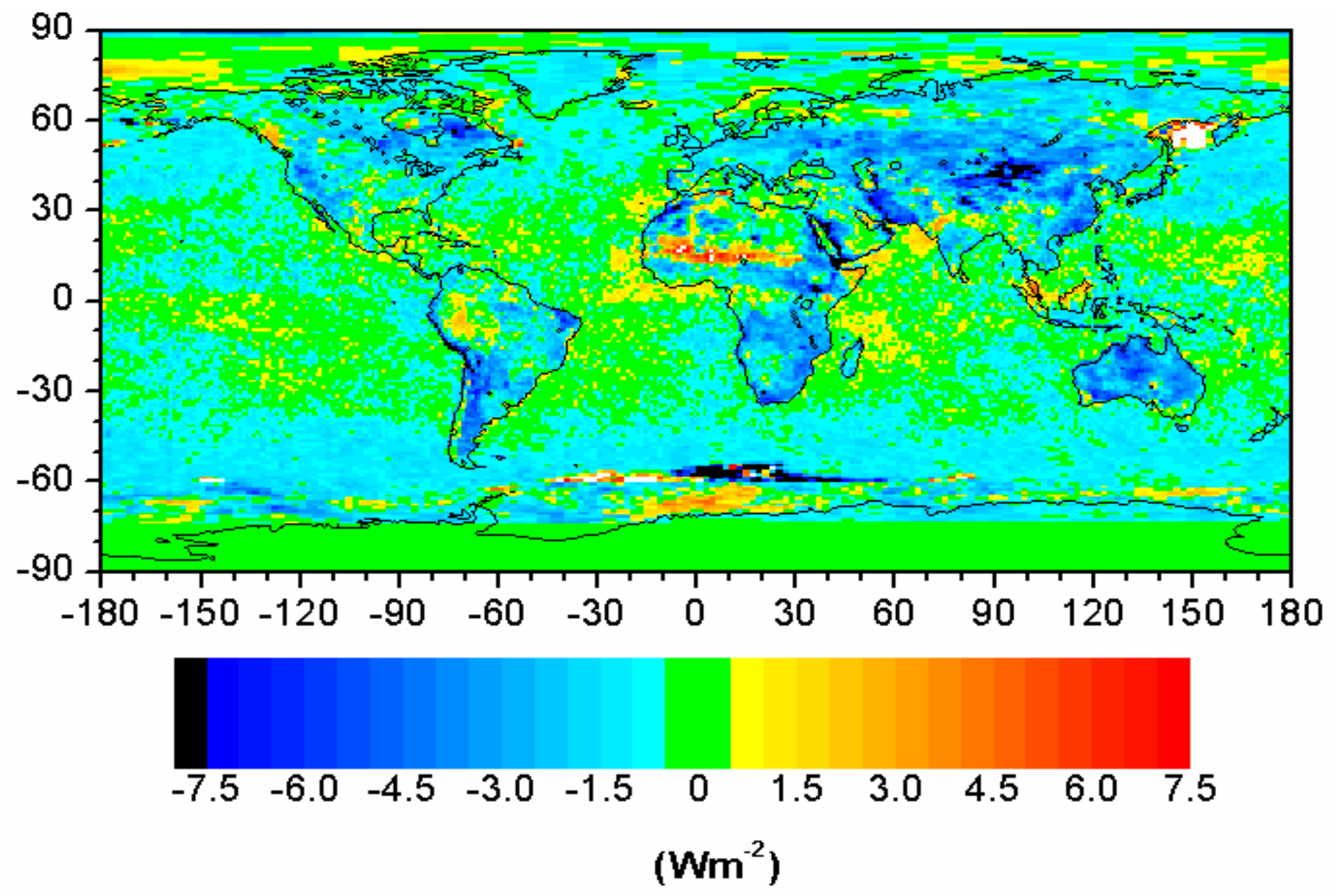

Figure 2 Change in regional annual mean SW TOA flux distribution between 2004 and 2001 based on the RTM approach with that based on radiative fluxes from CERES SSF product. 


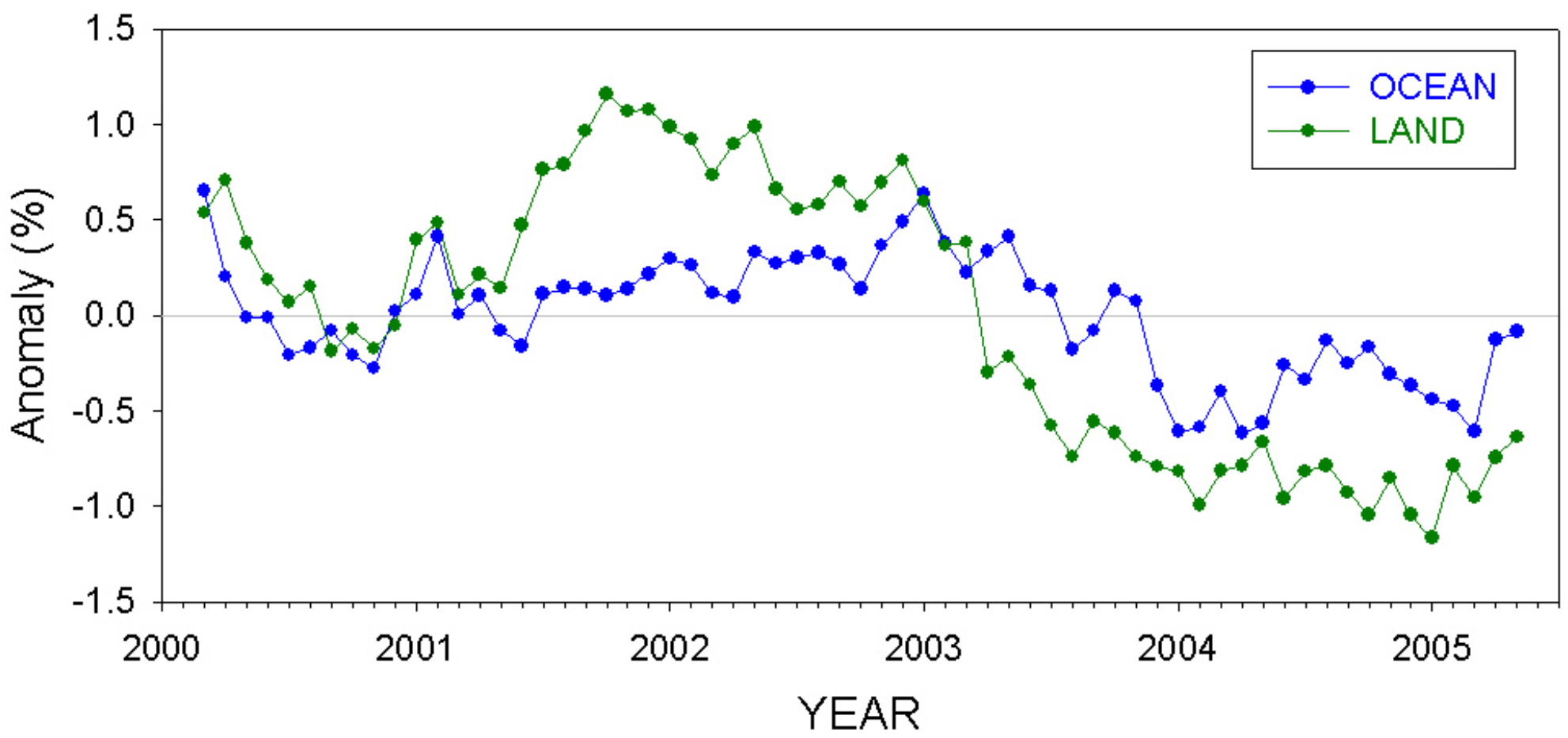

Figure 3 Deseasonalized anomalies in the relative difference between CRS and SSF reflected SW TOA flux for global ocean and land from CERES and MODIS Terra observations. 


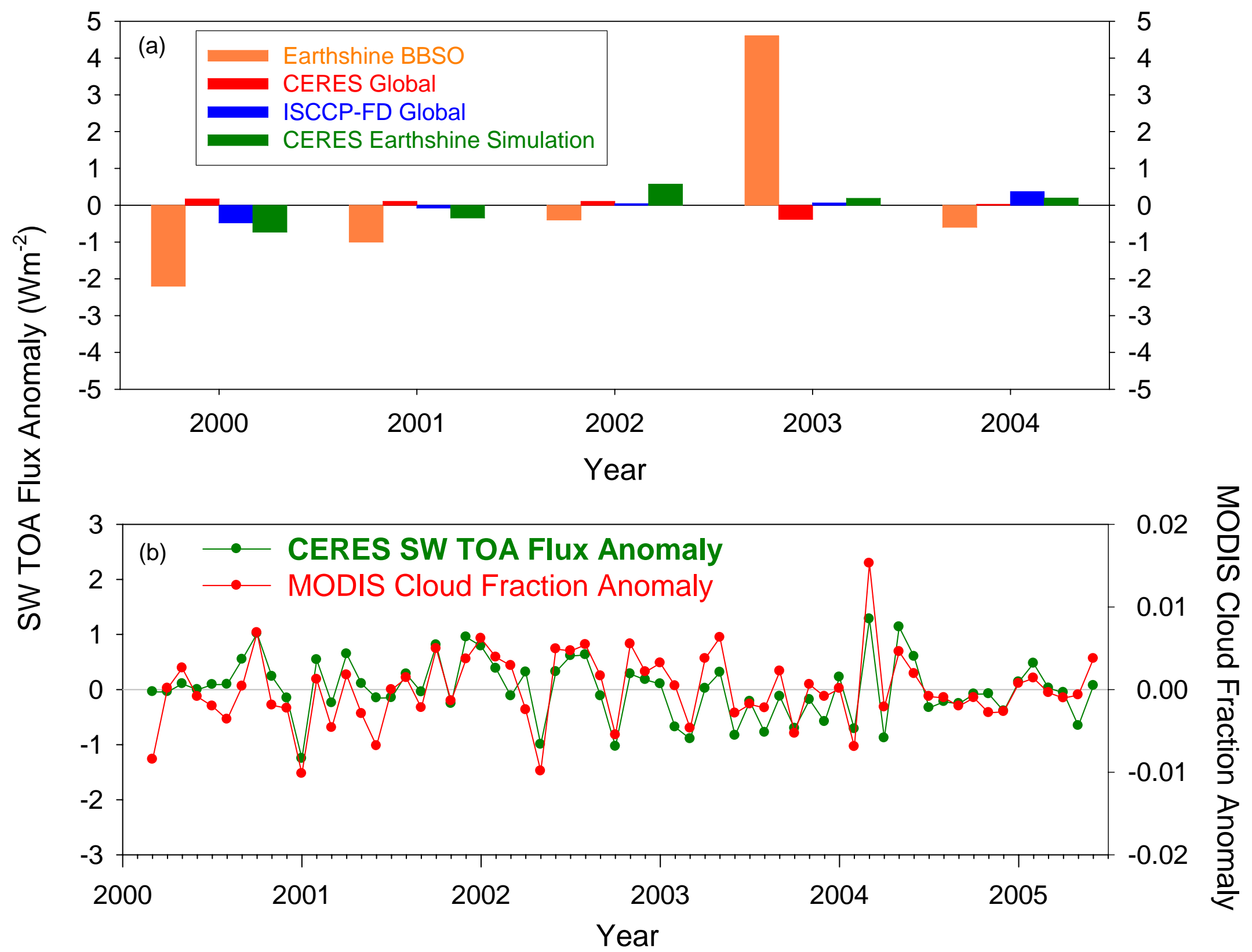

Figure 4 (a) Global annual mean SW TOA flux anomalies from Earthshine BBSO (Palle et al., 2005), CERES Terra global fluxes, ISCCP-FD, and a simulation of Earthshine. (b) Monthly anomalies in global mean CERES SW TOA flux and MODIS cloud fraction. 